\title{
A Jigsaw Puzzle As Model For Macroeconomic Analysis
}

\author{
José Villacís, (E-mail: villacis.fhm@ceu.es), Universidad CEU San Pablo, Spain
}

\begin{abstract}
A particular approach to the macroeconomic theory of production is considering production as a jigsaw puzzle that must be approached from a combinatorial point of view. This implies that an end production for the entrepreneur is one of the various possible ways of achieving production, and one particular mode is the optimal one-the one that provides the highest production at the lowest cost. Production factors can be combined or ordered in various possible ways and each combination will deliver a different level of production. Each combination in production is called a menu. Therefore, a jigsaw puzzle can be understood as modelling the necessary steps in the selection of menus to achieve the final creation which is the final production. Working with the jigsaw puzzle approach will require the task of numbering and counting all menus, assessing them, putting them in sequence and, eventually, selecting the best of them. The total and singular reality of a jigsaw puzzle will be the highest production that has been achieved with a specific combination of factors.
\end{abstract}

\section{INTRODUCTION}

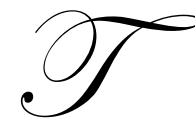

he production rate depends on the rate of consumption of factors, characteristics of the product and technology. This paper underlines an essential feature: combinations, the way goods or factors are placed in the sequence of consumption and production. Therefore, the combinatorial theory is included in the macroeconomic analysis that is here focused on the theory of production.

The concept of combination refers to sequence and placing of the elements. In consumption and production it is possible to order the goods and factors in different ways, as many as possible, to obtain different utility and production levels. Each combination of goods and factors will necessarily determine different levels of utility and production although it is possible, hardly possible, that indifferent combinations might exist - where different combinations bring forth the same production. This paper is based on the hypothesis that there are never two or more orderings that determine the same production level.

This paper only deals with the theory of production. Production factors are included in their simple numerical expression, regardless of their technology and nature. They are arranged in the different combinations. Each combination is called a menu in production and each menu will generate a different production rate and, therefore, there will be as many menus as possible combinations of production factors.

A puzzle is a fragmented reality. The building of a jigsaw puzzle consists of the necessary sequential steps to put the pieces in order and build reality as a unit. In the theory of production, in its combinatorial dimension, the steps to be followed are:

- $\quad$ Making all the possible menus or combinations and,

- Secondly, selecting menus from the best to the worst; in other words, eliminating those that are not the optimal ones.

Each step will imply a certain energy consumption that will be some quantum and its amount will be the cost of building the jigsaw puzzle. 
The first idea is that there will be a level of total cost measured by the addition of all the possible combinations, be it the cost of making all menus, selecting the best and eliminating the inferior. This statement is an understandable mistake. However, it cannot be admitted since the velocity in the integrating procedure is not constant.

There is some acceleration or increase in velocity as the puzzle building progresses. This is a manifestation of what is happening in the practice of production in a company's economy.

A jigsaw puzzle is a game with a single player but no dynamic strategic opponents. In this particular theory of games, there is a player working on a single material reality that is a jigsaw and, therefore, different to a card game or chess, there is not any dynamic change in reality. This is an important issue to clarify.

On the other hand, there are several types of jigsaws or games. One of them is the jigsaw consisting in a map (landscape or drawing, etc) divided into irregular pieces to arrange until the landscape appears. There are also crosswords and numerical jigsaw puzzles as well. The former, the map, is difficult to make at first and it becomes increasingly easy, as the building progresses. This is the kind of jigsaw puzzle this paper deals with. It is a working model. Games or jigsaw puzzles where the difficulty increases as they are being built are excluded from this model.

\section{WHAT IS A MENU?}

An entrepreneur has a series of production factors $(a, b, c, \ldots)$ to turn into production and reach a production rate. Combining, for him, means the arrangement in a group of some elements belonging to a series. It is a concept originating totally from the combinatorial mathematical theory and includes various partial approaches in mathematics such as permutations, variations, etc. It can be theoretically applied to the consumption and production theory, but it has serious restrictions in the theory of production.

The concept of combination does not imply any problem in the theory of consumption but it does in the theory of production considering the nature of the productive process. Each production implies a specific way or production process that, at the same time, determines a more or less fixed combination. In these circumstances, the factor combination possibilities are seriously limited. For instance, a farmer cannot plant its seeds if the land has not been ploughed previously. A cook will necessarily take a pan, pour in some oil, ignite a fire, and then fry the egg, and this order cannot be altered. As a consequence, the possibility of combination will only happen in a limited band that will be called possible combination band.

What is the meaning of combination? Combinations are all possible ways of placing, or better, arranging all elements, in this case existing production factors, with no repetitions. It means that all elements (all factors) are present and all possible groupings are manifested, with no repetitions. This is a singular case of the combinatorial theory called ordinary permutations. Accepting this mathematical concept, every combination of production factors is a unique menu.

To start, a batch or universal menu will be the group of all production factors available to the company for production. And, considering the combinatorial dimension, in this case, each ordinary permutation, each specific group, will be called a menu. Therefore, the company can create several menus given the number of elements or production factors and the several forms of ordering or combining them. Once all the menus have been established, the company will choose the one providing the highest production rate supposing there are not two or more equivalent combinations as far as the production level is concerned. The menu providing the highest production rate is the optimal menu and, therefore, the inventory or numbering of all menus is a necessary requirement for choosing the optimal menu, the most convenient one for the company. These menus are called specific menus.

But, as it has already been mentioned, not all menus play and the non-playing menus are not considered in the choice of the optimal menu-only those technically feasible come under consideration. This means that technically impossible menus are rejected. 
Impossible or irrational combinations or menus are rejected. Therefore, if the elements or production factors are $\mathrm{n}$ and they create a group of combinations or ordinary permutations, the actual or possible permutations will be in fact lower than those that can be mathematically considered, since a group of them will be rejected.

\section{SYNTAX AND COUNTING POSSIBILITIES}

A jigsaw puzzle is made of a precise number of pieces. None of them is left over, none of them is missing. Everyone is necessary and indispensable. The opposite statement is valid: the addition of all pieces will form the total area and the logical unity of the jigsaw puzzle. This implies two complementary realities: the first one is the simple fact that all the elements belong to a unit; and the second one is that this unit implies that they are logically placed in the group; something like a single language formed by exact words linked by syntax.

Therefore, the next steps should be followed: enumerating the pieces in the jigsaw puzzle and then placing them in the group. It should be observed that they are not two different operations, but a single, continuous and eliminatory activity. An inventory must then be made - the purpose of this section - of all pieces belonging to the jigsaw puzzle and it has to be explained what a puzzle is in the combinatorial dimension. Several possible combinations can be made with all the pieces in a jigsaw puzzle, but only one of them will be valid, the rest being illogical or meaningless. The issue to be considered is the combination of the elements in the jigsaw puzzle offering the highest possible production and this implies a specific combination, and not any other, of the production factors. There will be some other combinations of production factors not found in the jigsaw puzzle which are not technically possible either.

The exact number of pieces is the total number of all production factors, when none of them is left over nor missing, and the logical unit mentioned is the specific combination that determines the highest production level. This would be the logical unit of the jigsaw puzzle. This is the process of each production economically and technically speaking, the syntax that makes sense of the factors combination. Once the n number of pieces or production factors is known, the number of possible combinations has to be counted to choose the one which provides the highest production level among them. This is the most appropriate syntax.

Combinatorial mathematics works on counting operations related to combinations of the elements in a group. In combinatorial mathematics, it is ordinary permutations that make it possible to count all the groups where all elements are included but no one is repeated. It means that all the production factors in a process are included but none of the factors is repeated and all these factors take part. If there are $n$ factors, the number of all possible groups is calculated with the following formula:

$\mathrm{P}_{\mathrm{n}}=\mathrm{n} !$

If there are, for instance six production factors, $(n=6)$, all the possible groups would be: 6.5.4.3.2.1=720. Among all possible groups (n!) called combinations in an intuitive way, there will be only one that creates a logical unit in the jigsaw puzzle, and the rest should be rejected because their choice is illogical. Outside of this order, in a specific combination or order neither the jigsaw puzzle nor syntax can possibly exist. This statement is only partially correct. However, some rigidities are considerably limiting it. It has been considered that each combination or order, or each menu, generates a single production level.

\section{POSSIBLE BANDS IN COMBINATIONS AND ABSURD COMBINATIONS}

All production factors on the one hand and, on the other, all possible $n$ ! groups have to be considered in the choice of the optimal menu. There are two successive and complementary inventories. Reality shows that in most production factors, there is a natural order in the sequences of production (mineral extraction and then melting, etc...). This is the possible band of combinations, and the remaining are the not possible or not logical or, if we want, impossible. An impossible band is formed by the group of combinations (among ordinary permutations) with which it is not possible to obtain production. They are not merely illogical combinations generating production rates not as high as possible, but no level of production at all can be obtained from them because they lack any meaning. 
These impossible bands in production are called absurd combinations. An absurd combination is the one that is not essentially operative. It is difficult to find an example of production where absurd combinations do not exist. These are intuitive and, obviously, discarded in production. The remaining combinations or groups belong to the possible band of combinations and, within that band, or subgroup of ordinary permutations there is an optimal one. Therefore, the complete jigsaw puzzle will be found within the band of possible combinations.

Two different groups can be considered: $\mathrm{A}$ and $\mathrm{A}^{\prime}$ where the former is the simple mathematical and theoretical numbering of all the combinations of all factors where none of them is repeated. This is the universal menu mentioned in previous sections. It represents the potential order of all production factors and its value is represented by $\mathrm{n}$ ! The second is $\mathrm{A}^{\prime}$. It is a subgroup in ordinary permutations, excluding absurd combinations. In this way, A' represents the real numerical or effective value of effective rational combinations in production and, as absurd combinations have already been eliminated, its absolute numerical value will be lower than ordinary permutations or universal menu: $\mathrm{A}^{\prime}<\mathrm{A}-$

The jigsaw puzzle is by definition formed by a combination maximizing production, and a combination that is, above all, possible. It is essentially rational. Therefore, the jigsaw puzzle is an order included in the group A'. There will be two successive eliminations in the jigsaw puzzle: the first one will be rejecting absurd combinations to reach the rational group A' that is possible from the point of view of production. It would not make any sense to make a jigsaw puzzle based on absurd combinations. Next, the non-optimal combinations will also be eliminated. They are not absurd but they are not rationally productive either.

A remark should be added here: It has been observed that a rational and possible jigsaw puzzle records combinations in a lower variety and number of elements than those that theoretical and mathematical calculations allow ( $\mathrm{n}$ !). This statement should not lead to the conclusion that a lower variety of combinations is due to the fact that fewer elements are included. The elements - in this case production factors - will be the same (and present in the same number). The universe of their combinations decreases and, more precisely, it decreases by the number of absurd combinations.

\section{RANDOM AND NECESSITY}

The question whether it is possible to build a jigsaw puzzle at random remains unanswered. To answer it, one must know the possibility of building a jigsaw puzzle by following the unpredictable paths of randomness. And the first thing to be known is all the possible combinations that could be established and the one, out of them, which is unique. There is a specific combination of pieces in a jigsaw puzzle: one single place for each piece and only one. This creates an order or interpretative syntax defining a map. In the case this paper deals with, each production factor is placed in a specific order generating the highest production rate. The pieces (factors), the order and the highest production rates are the jigsaw puzzle.

The total number of all possible combinations is $\mathrm{n}$ ! and they belong to $\mathrm{A}^{\prime}$, that is the rational group from which all absurd combinations have been excluded. So there are $n$ ! possible combinations. Therefore, if all pieces are thrown into the air, and they fall in perfect order, thus defining the jigsaw puzzle, the probability of this happening is measured by:

Probability $=1 / \mathrm{n}$ ! would be:

On the contrary, the probability that the puzzle would not be made at random, but rational and voluntarily,

$\mathrm{n} ! / \mathrm{n} !=1$

Therefore:

$1>1 / \mathrm{n}$ ! 

at random.

The conclusion is that it is always preferable to build a jigsaw puzzle rationally than leaving it to fall in place

There is only one case where the outcome is indifferent to building a jigsaw puzzle or not building it. This case happens when there is only one element. As there is only one element or factor, there will not be any possible combination and neither random nor rational activity makes sense and production, whatever it is, becomes unique.

It is possible to widen this research to the field of consumers and include it with the theory of production. This widening would put some strain on the method but it should be done. The problem is as follows: Building a jigsaw puzzle rationally requires an effort in various ways: making all possible combinations (permutations) and the selection of the optimal combination. This task is effort-consuming or generates disutility; it allows obtaining an optimal rate for production that will be consumed by consumers. This consumption will generate utilities in the sense of the highest proportion generated in production. In this way, with this reasoning, building a jigsaw puzzle is always interesting.

\section{ENDOGENOUS INFORMATION}

Implicit endogenous information is underlying when dealing with the information on combinations. Production factors are not equally efficient in production if they are considered individually. For instance, in agriculture, water is essential and a certain type of fertilizer, phosphates, for instance, may not be equally efficient in production. The following question then rises: Will the optimal combination necessarily show that factors will be applied in a decreasing order? The answer is: not necessarily. It depends on the nature of the production process and the technique. The place or order of the elements to make the jigsaw puzzle is an empirical issue rather than theoretical or mathematical. The place of each factor, putting the most efficient one in the third place, for instance, will make it more efficient than if it were in the first place. The principle of decreasing marginal productivity seen from a general point of view is not always accomplished.

Each combination transfers its own endogenous information to the production system. This information is different to the one of each factor, of each production process, and to the technique. All this is information determined from outside the system. At the same time, the successive changes in order will generate theoretical changes in production volumes and nature, according to that endogenous information.

\section{THE SCALE OF MARGINAL PRODUCTIVITIES}

Combinations or orders in the context of ordinary permutations, in spite of their exact mathematical and economic meaning, can be conceptually flexible to include other interpretations rich in content. Some approaches will enrich the general idea of marginal productivity. One of them is making incomplete combinations with the structure of optimal orders. The other one consists in the comparison of non-optimal but gradual orders from the worst to the best, as a scale, including all factors in production.

In the first case, factors are used and combined but not totally. Then, the remaining factors will be added and they will also be combined. Partial menus are created, that are the partial orders of a part of the optimal menu. This way of considering combinations is temporal or sequential until the total time-period comes to an end with the building of the jigsaw puzzle. Variations in marginal productivities can be analyzed by admitting the successive inclusion of new factors in the general plan of optimal sub combinations. This consideration is the same as for the traditional of marginal productivities. In this pattern it is still possible to analyse increases in total productivity and even marginal productivity, in this case from the combinatorial dimension only. It means that if new incoming factors are not being incorporated in an optimal way increases in production are then frozen. As a consequence of the redeployment of such initial production factors, more efficient combinations are made, until the optimal combinations are reached and total productivity increases.

In other words, the producer now orders the arriving production factors and their arrangement in better ways when measured in terms of production. This causes an increase in production. These increases in production create an 
upward scale in production from a lower to a higher region, going through intermediate steps. The lower steps, once a layer of production factors has been established, constitute suboptimal combinations and, as these orders improve, it is possible to ascend to the top, the highest production. This production level is relative. It is not the jigsaw puzzle as a whole but a part of it, because not all factors have been included. According to these points, there will be suboptimal combinations with some production factors that are not complete - but only a part of the total. These have been called incomplete combinations.

What can be said about marginal productivities? Is there the same vertical distance among all the steps? Nothing can be categorically concluded about general productivities if orders are exclusively isolated. They may grow more than proportionally at first, with the new orders and then, as they approach the optimal sub combination, their growth might become slower or less than proportional. But these conclusions are nothing but intuition when considered apart from empirical facts. Above all, they depend on the nature of each type of production.

If time and evolution are considered in the reasoning of optimal sub-combinations and the successive inclusion of factors is accepted, the terms change. In the first stages of any production there is usually decreasing scale yield due to the better coordination of production factors, the freshness of the early productive impact, etc. If the fact that the entrepreneur also arranges or combines these factors in a better way, it may be concluded that marginal productivity increases: the steps in the ladder are higher (further apart) at first. Then tiredness and the lack of coordination among factors occur. Saturation makes marginal productivity decrease. And, in that area of production, the best order or combination stops taking place and they cannot neutralise the decrease of marginal productivities. This is the explanation of non-total orders of factors or sub optimal sub combinations.

The second consideration is that in building a jigsaw puzzle, all factors are included. The jigsaw puzzle is made by using successive combinations until the optimal one is reached: the optimal menu. Each combination will imply certain production rates that will be ranked from the lowest to the highest one as in a ladder. At the end of it will be the optimal menu, the one providing the highest possible production rate.

Marginal productivities must be analysed in this context. They are really possible because all production factors are involved, as well as all possible orders defined by ordinary permutations. In the example of the ladder, each step contains all factors ordered in a specific way. Marginal productivities are measured by the difference between two successive productions, which means that they are determined by the efficiency in production of two successive orders (two successive steps).

\section{THE MULTIPLYING JIGSAW PUZZLE}

We know that the more pieces in a jigsaw puzzle, the more the effort of assembling it. The fact that one jigsaw puzzle has more pieces than another does not mean that its physical and numerical dimension will necessarily be proportional or proportionally bigger than the other one.

A simple case can be noted: when each piece in the jigsaw puzzle is split into equal pieces forming, in that way, a jigsaw puzzle with more pieces than the original model. Seen from that point of view, it can be explained in a different way: a jigsaw puzzle formed by $\mathrm{k}$ times the $\mathrm{n}$ original pieces. If $\mathrm{n}$ is the number of pieces $(\mathrm{a}, \mathrm{b}, \mathrm{c}, \mathrm{d} \ldots)$ and each piece is repeated $\mathrm{k}$ times, the number of pieces equals k.n. Ordinary permutations determine that the possibilities of ordering them are $\mathrm{kn}$ !

Then the question arises whether the effort of building a jigsaw puzzle should be measured by kn! times. The answer is negative as far as each fraction of each piece is a numerical, syntactical, or logical equivalent to the main piece. An example could be a kilo of fertiliser in portions of 100 grams. This reality must not lead to believe that there will be 100 new realities to be ordered or combined.

The first reasoning is that if each piece is considered as formed by a series of pieces that are sub multiples or the original one, all those pieces can be considered as a whole that would be that factor or element. And the building 
of that original piece would only be the addition of the partial ones and not the addition of combinations or possible different orders. Therefore, the addition of pieces will always be less than all possible orders.

$\mathrm{n}+(\mathrm{n}-1)+(\mathrm{n}-2)+\ldots .<\mathrm{n} !$

Reason and intuition lead to different ways of understanding it. If each piece is repeated $\mathrm{k}$ number of times, the placement of each fragment in the combination will automatically determine the situation of the remaining pieces. Therefore, the total building of the jigsaw puzzle requires less effort.

There is a remarkable difference between building a jigsaw puzzle with $\mathrm{n}$ pieces and building another one with kn pieces. In the second example shown, each piece is formed or repeated by partial pieces easier to put in order than in the first instance. It is the same repeated element what provides a considerable advantage to the assembler.

\section{WELDINGS AND HARD CORES}

It is possible that in a jigsaw puzzle the pieces or factors - elements, in mathematics -could be welded. Weldings are specific sub combinations among production factors that are relevant because of their efficiency or necessity in production. If this sub combination is technically preferred, it becomes indispensable in any productive process. Producers will be more interested in this sub combination, than in any other combination or general order where all elements are involved. This sub group will make them behave as a single block. It will be possible to break the welding and free the elements to be combined in different ways, but it will not represent the highest possible or efficient production rate. It means that weldings do not imply that it is not possible to combine factors in a sub combination in other different ways. It is possible. However, it would not be an optimising sub combination.

When this welding groups some production factors, this group will behave as a single piece or factor. This special group is no longer offered as a menu of factors that can be combined since, technically and by definition, it is welded, in such a way that the combinatory effects of that group of factors are not considered in the combination. It would be the same as stating that the jigsaw puzzle has got fewer pieces without losing size or integrity and the effort (the complexity) to make it have decreased.

Following this reasoning it can be concluded that the number of possible orders is not $n$ elements, but less. There will be an $\mathrm{m}$ group of factors kidnapped in weldings and, therefore, they cannot take part in combinations freely but, on the other hand, they do take part as elements of a group in weldings. To ascertain the number of possible combinations that determine the jigsaw puzzle, the number of $n$ factors will be reduced in an $m$ amount - those belonging to the welding but, as weldings take part as a unit, the combinatorial game should also include the number of such weldings. If this number is n', the total number of elements involved in building a jigsaw puzzle will be:

$\left(\mathrm{n}-\mathrm{m}+\mathrm{n}^{\prime}\right)$

This figure is lower than the initial $\mathrm{n}$.

$\left(n-m+n^{\prime}\right)<n$

The amount of possible orders will be:

$\left(n-m+n^{\prime}\right)$ !

It can be concluded that the number of pieces in combinations would be higher if weldings did not exist than if they did. The reason is that weldings comprise several production factors in the same group.

And the last remark: Weldings will not always follow the criteria of higher production. There are other criteria influencing the creation of solidly linked combinations, such as commercial or factor provision, institutional as 
the formation of trade unions, etc. They may even be a way to make combinations easier for producers. In other words, the formation of weldings can be a strategy to save energy when building a jigsaw puzzle.

A special case of welding is the hard core. It is a sub combination that links factors such a technically intense way that it is impossible to replace by any other order. Its difference from the others is that those allow different orders that imply a decrease in productivity. Besides, in a negotiation among producers they are negotiable or can be subject to cessions, although with some difficulty. The hard core, however, is rigid and it is impossible to replace it by any other order. It can be neither negotiated nor subject to cession. The hard core is not subject to the wish of producers or jigsaw puzzle makers and is a strictly technical issue. The final jigsaw puzzle identified with the optimal menu is not a hard core because it is always possible to make other jigsaw puzzles that are not optimal. This is the essential difference with other types of combinations.

\section{THE COST OF BUILDING A JIGSAW PUZZLE}

It can be considered that a movement, whatever it is, in building a jigsaw puzzle consumes a unit of energy called quantum. In building a jigsaw puzzle, the unit of movement consists in placing each factor in the group of a specific order, in such a way that any number of combinations, 720 for instance, will imply the consumption of 720 units of quantum. Therefore, there will be as many orders as units of quantum because each placement or order of a production factor implies the consumption of a unit of energy or quantum. The shift from the position $n-1$ of a factor to the position $\mathrm{n}$ will imply consumption of energy and in the example above it would mean that in the case of six factors, the shift from the position 719 to 720 would mean consumption of a quantum unit of energy.

This information makes it possible to assess the total cost derived from building the optimal puzzle, determined by the total addition of units of energy resulting from building all possible orderings without repeating any factor (ordinary permutations). This addition would be reflected by the formula $P_{n}=n$ ! In the example above, it refers to six factors: $6 !=6 \cdot 5 \cdot 4 \cdot 3 \cdot 2 \cdot 1=720$.

It is estimated that the unit cost of ordering equals the marginal cost and, therefore, the marginal cost (of all and each step) is always equal. Seen from this point of view, the evolution of the total cost is rising and linear. These conclusions are reached considering the theory of order shown before and the strict hypothesis that each unit of energy is worth a quantum. Besides, the position of each factor will consume a unit of energy. This statement will be altered in the following sections.

\section{ECONOMIES OF SCALE IN BUILDING A JIGSAW PUZZLE}

The existence of a quantum of energy worth a unit does not mean that it cannot be subdivided into submultiples. This simple remark will be useful to understand the following concepts related to the true reality of building a jigsaw puzzle.

The truth is that, in spite of the points that have been made in the previous section, the position of each production factor in an order is not equal to the energy consumption of one unit of quantum. In a traditional jigsaw puzzle, the first movement is more difficult and intuitive and implies higher energy consumption. Following an extremely simplified model, the first piece implies a higher cost; the second one is less costly and so on. As the building of the jigsaw puzzle develops, the speed increases until it is suddenly stopped when the jigsaw puzzle is finished.

This reasoning establishes several energetic criteria, that are not much different in nature: it can be accepted that each of the initial movements consume a multiple of a quantum, then, there will be one in the series consuming one quantum and, since that piece is put in its position, the following ones will be consuming fractions of a quantum.

Another criterion can also be established, which is not very different from the previous one: it can be assumed that the first movement or the first piece consumes a unit of quantum and the successive ones will consume fractions of a quantum. The result is the same. What is important to understand is that, in a traditional jigsaw puzzle, 
the cost of a unit in building it is successively decreasing. It is possible to measure the total cost of a decreasing geometrical progression $\mathrm{r}$, where the first factor is worth a unit. This addition is expressed as follows:

$\mathrm{S}=1 / 1-\mathrm{r}$

It can be noted that a remarkable contradiction exists between the starting points in the previous section and this one. In the previous section it was stated that each unit of movement in each order (placing each piece in the jigsaw puzzle) would consume exactly a unit of energy, which affects all and each of the movement's unit and marginal costs. All of them consume the same amount of energy: one quantum. In the second section, which is based on reality, each movement of each placement of a piece follows a decreasing path. The formula measuring the total cost of building a jigsaw puzzle as $\mathrm{n}$ ! is no longer valid.

Why has this contradiction been used in the research? It has been applied to underline a very important feature in Microeconomics: the existence of economies of scale in the theory of production. Economies of scale in building a jigsaw puzzle are measured by the difference among the numerical values of all orders, assuming that the energy costs of each $\mathrm{n}$ ! order is constant, and the total costs - admitting the successive reduction of unit costs 1/1-r. This difference: $n !-1 / 1-r$ develops the advantages or economies of scale taking place in a rational and intuitive way.

It is still possible to develop another reason based on the speed in building a jigsaw puzzle that confirms the existence of economies of scale. As the building of the jigsaw puzzle progresses, skills and intuition in learning how to build it, increase. At this point, something possible is not considered: the reason of the decreasing geometrical progression may also increase.

\section{AGREEMENTS IN PRODUCTION}

\section{The General Case Of Agreements}

In the same way as it is possible to understand that two or more producers will join together to produce, it is possible that they join their efforts to build a jigsaw puzzle. The sense of cooperation can have several meanings: it may happen that some would contribute with the factors and others would put them in order. Another possibility would be that producers would perform both tasks: supply the factors and cooperate in their arrangement. The true cooperation in production takes place in the latter case.

In this cooperation, each group can offer its experience on its own production or, in other words, its experience on building their own jigsaw puzzles. Each group's experience on more restrictive orders provides more efficiency to the final building of the jigsaw puzzle as it is more specialised and, therefore, the agreements in production will generate higher economies of scale than if the same process was done individually.

A more complex issue can be observed. Is it possible to divide a jigsaw puzzle into two, three or four parts? Would this splitting make any sense if it were possible? It means that there are batches or groups of different orders and then, all these groups would not be put together until the final jigsaw puzzle is completed. The agreements among several producers mean in this case that each producer is specialised in a section of the final work. This specialisation is a consequence of their previous activity as independent producers and, because of this specialisation, intense economies of scale will occur.

In general, and due to specialisation, the agreements in building a jigsaw puzzle will generate economies of scale.

\section{Agreements With Weldings}

Each individual may establish his own philosophy on the efficiency of production. This means that all of them have to agree necessarily on the optimal order, with general rationality. There might be a more serious drawback on criteria of optimal sub-combinations, offered as a block and difficult to break. This means that each producer, 
before joining another producer to cooperate, may offer not only his experience on efficiency criteria but also factors that might be welded.

In the section dealing with weldings, it was said that weldings are orders, within a general order, closely linked by the features of production or by means of efficiency.

At this point, it will have to be stated that producers cannot offer their factors to be freely combined but can only offer factors welded and factors internally tied in weldings. The existence of weldings may be the origin of conflicts in cooperation for production. The other party may not be willing to accept the pack to make the jigsaw puzzle. The game, or better, the cooperation, requires free access to pieces or factors: the ability to order them freely. This does not mean that the parties involved may have weldings and those weldings may not coincide. Then, a pact or agreement about the order becomes necessary.

All of them will be interested in achieving the building of the jigsaw puzzle, and they will reject the idea of leaving it unfinished due to internal conflicts. We must then present the idea of a final prize that is the jigsaw puzzle defined by the optimal menu with the highest production rate following technical and order criteria. The final prize will be the appeal that will lead the parties to negotiation. Therefore, it leads the way to the optimal bribe that those who receive more benefit will try to use to compensate the others for their cooperation in putting the pieces in order. This way of thinking is the same as the following one: those who would loose if the building of the jigsaw puzzle would not take place would agree to cooperate on the condition of developing new arrangements. Weldings will be broken to negotiate. In fact, the negotiation will consist in breaking the weldings.

Two groups are considered to carry out production: a strong and a weak one, and two simultaneous cases too: one of them with weldings, but not the other one. In the first case, the strong group has strong weldings, but not the weak one. The strong group will feel very interested in finishing the jigsaw puzzle, with more intensity than the weak group. The strong group will value its weldings much more highly and, therefore, it will be ready to bribe the weak group to accept them. Such bribery will consist in giving a part of production that initially would correspond to the strong group. The acceptance of this bribery will allow weldings to be included. The process will take place and the velocity of building the jigsaw puzzle will increase because those pieces are considered as a block.

If the weak group (that is also obviously interested in building the jigsaw puzzle) has the weldings and not the strong group, it will be bribed by the strong group to discard weldings by offering them part of the final production.

The jigsaw puzzle represents the optimal menu that determines the highest production rate in such a way that all groups will be interested in achieving it, even weak groups. They get the smallest part of production and most of it is for strong groups, but all of them are interested in building the jigsaw puzzle, which makes them obtain the highest production rate. In this way, briberies will be successful in the first case and blackmail in the second one. They will always be willing to give up the weldings. Not doing so would mean not achieving the highest production rate. Strong groups will not be willing to dismantle their weldings and will be successful in bribing the weak groups since those will receive the compensation of a portion of the highest possible production rate. In the second case, blackmail will imply the usurpation of a part of the highest final production and therefore, they will be willing to accept renouncing their weldings.

It must be noticed that there is no pact or negotiation, neither bribery nor blackmail in the case of the existence of hard cores. In fact, all the parties involved consider them as a single datum; that is to say, as a block of strongly welded pieces whose order is impossible to change. The existence of hard cores shortens the negotiation, as it is a part that is never included in negotiations and, on the other hand, makes the final construction easier. Why makes it easier?

The existence of hard cores shortens negotiations, as it is a field that is not included in negotiations and makes it easier to create the jigsaw puzzle. Why does it make it easier to create the jigsaw puzzle? Because, as in the case of weldings, these sub-orders of factors are considered as macro pieces that gather together a group of pieces, and 
then the total number of pieces will be lower than the simple addition of $\mathrm{n}$ factors. It has already been noticed that $(\mathrm{n}$ $\left.m+n^{\prime}\right)<n$. And, as there are fewer pieces to be put in order, the building of this jigsaw puzzle is faster and, therefore, less costly.

\section{THE COMMON JIGSAW PUZZLE AND OTHERS}

There is only one way to place pieces in any jigsaw puzzle and, therefore, there is only one map defining a jigsaw puzzle. In our combinatorial approach there are many ways (n!) of arranging the pieces and there will be many different jigsaw puzzles defining various production rates. Those different production rates can be measured quantitatively and they can also be arranged according to ordinal preference criteria.

Each jigsaw puzzle will have the same number of pieces and factors and the same number of combinations, as far as there are neither weldings nor hard cores, even if their size is different. This means that not all of them will generate the same production rate if the hypothesis that two or more do not show the same production rate is accepted. If all jigsaw puzzles are measured by $n$ ! and only one of them is the optimal one, the remaining number, more precisely $n$ !- 1 will be suboptimal. In cases where there are weldings or hard cores, as it has been observed, there will be less different production rates, that is to say, less jigsaw puzzle variety because there will be less possibilities in combinations.

The existence of weldings and hard cores frustrates the exact knowledge of the number of pieces or total menus. If several pieces are grouped in weldings, it is not possible to enumerate all possible combinations since they are not included in orders. However, the existence of weldings or hard cores does not frustrate the knowledge of the jigsaw puzzle or optimal menu, since weldings imply a specific order (sub-combination) that is technically efficient by definition. This statement is categorical in the hard core. It is impossible to alter this order from the technical point of view. It can be concluded that many jigsaw puzzles can be made but only one will be the optimal, or representative of most efficient production. The existence of weldings and hard cores reduces the number of variations in order of building jigsaw puzzles, but they do not prevent the definition that the jigsaw puzzle obtained is the optimal one or optimal menu.

\section{ASYMMETRICAL INFORMATION AND ADVERSE SELECTION}

In order to meet the requirement to make a jigsaw puzzle successfully it is necessary to have exhaustive information on the following issues:

- All and each of the factors, their nature and influence on the end production or their marginal productivity.

- $\quad$ The influence in the production of each specific order.

- $\quad$ The influence of the map that is going to be created.

- $\quad$ The influence on the technique.

All this information should be known by the player or players assembling the jigsaw puzzle. The market supplies the information about different issues such as: supply, prices, demand and, over all, on the performance of suppliers. It has to be noticed that players will always try to obtain the most complete information. They are interested in obtaining the highest production rate in exchange for the optimal jigsaw puzzle.

However, the fact that comprehensive information must be obtained does not imply that it will be obtained. It won't, probably, because of irrational behaviour in the market. If it is assumed, for instance, that factors and information suppliers in the market would hide information or would offer misleading or insufficient information, asymmetrical information would reach the market and its consequence would be moral risk. This is the case when one of the agents in the market is provided with more information than others. There are two different manifestations of our ordering: one of them is the hidden action and the other one is the hidden feature. 
In the first case it is possible that a person, a producer in general who is the major producer, may ask someone else, an agent, to make the jigsaw puzzle. In the second case, it would be a seller of factors who knows the nature of the pieces better than the producer. This paper will deal basically with the first case.

The behaviour or relationship between the major producer and the agent will be studied bearing in mind that the latter enjoys an information advantage over the major producer, and this causes asymmetrical information. The agent, who is in charge of building the jigsaw puzzle, will have more information that his patron in two ways. The agent will know productivity better and the reasons for his own efforts and, on the other hand, he will know more about the efficiency of each of the arrangements that the puzzle may have.

This imbalance in information will cause the moral risk since it encourages the agent to act under its own real possibilities. That is, to avoid his responsibilities thus deceiving the major producer that pays him. A series of interactions between the agent and the major producer take place to achieve a balance or neutralise each other and let the market work efficiently. The moral risks will then decrease or disappear. In the end, the major producer is also aware that he is facing unequal conditions and will set up a series of compensating measures, to avoid moral risk.

One of these measures is to increase control, an activity that increases the cost per unit and the total cost of production. Another measure is to increase salaries paid for ordering factors to dissuade agents from behaving inappropriately, and rather make them behave more carefully. And the last measure, the most efficient one, will be paying workers according to their production, once such production has been successfully completed. This measure is adequate in the example studied in this paper because it forces the agent to act efficiently because its final payment, in the end, will be determined by the efficiency, intelligence and speed used to put the factors in order and complete the jigsaw puzzle. The producer - in this case the major producer - can also adopt all kinds of compensating measures.

Adverse selection appears in markets when a perfect competence in the market of information does not exist and, more precisely, on the nature of the item to be sold. In adverse selection, the seller knows the nature of the good to be sold better than the buyer. Then, there is a potential prejudice impacting on the misinformed party because it does not have enough information on the good, to the point of thinking that the good is not desirable. The good they try to sell in this case is the work factor and, therefore, what it yields in such a way that workers are better informed about their skills than the seller entrepreneur.

The entrepreneur pays workers a salary to combine factors and even to check the quality of the pieces or factors in the order. It will have to be discovered which is the market of workers who arrange factors in order (or the combiners) and determine their price or salary. This salary, even in the case of a perfectly functioning market, is not the perfect solution or omni-comprehensive one that guarantees an efficient resource allocation: The best production (order) with the least possible effort.

The reason is that, when information is imbalanced - when information is asymmetric - productivity is not reflected in the salary. To avoid this asymmetry or to compensate for it, the entrepreneur will pay its workers who arrange factors in order a salary higher that the one offered on the market. This is an approach, a point of view, and a solution that does not explain this complex issue in a final way.

Selection, in the context of adverse selection, is the action of the misinformed party to create signs for the informed party and compensate information from both sides. A way to create signs for workers will be establishing potential wage differences according to end production; that is, to offer a reduced wage if the work is not finished in the time period and way established in the contract, and increasing the salary above the level established by the market in the case it is finished satisfactorily. The selection and signs will try to place the necessary information in the market for an efficient allocation of resources.

The intervention of public powers could be justified in this context. But public powers are a general and undefined term that can be replaced by Leviathan, which is more operative and precise. Leviathan can distribute information or force information to appear in a more fluent way and decrease asymmetric information. But this example is not always desirable because Leviathan, by definition, represents the power of political and social groups 
that use power. If people trust the State or the public powers nowadays to publicise information is it because there is a tacit agreement in society that the State is the protective father who will always supply suitable information? It will only be an emotional and cultural criterion.

In essence, Leviathan can be defined as a strong force resulting from partial vectorial forces defined by political and social groups that act according to partial and selfish interests, the same as individuals in a private economy. This statement means that Leviathan can become a source of asymmetrical information. If this were not the case, then there are not many instances where the state has access to more information than individuals and its participation is not totally justified. In building a jigsaw puzzle, Leviathan's participation to inform the entrepreneur on the criteria of workers can even be counterproductive. This information can even be partial or irrelevant in the ordering process.

\section{NATIONAL PRODUCTION}

The issue of order can be focused on from the point of view of domestic production or gross domestic product. It is considered as a suitable methodological step from microeconomics to macroeconomics.

Domestic production can be measured as the total added value by period, and conclusions can be drawn from this definition. Each increase in production or each added value represents the total of partial added values. It can be reduced to each portion of the latest production and estimate that the total by period determines the partial added value in each period and, at the same time, the addition of all of them is the domestic production.

Each last portion will be called a unit production of added value in the smallest possible jigsaw puzzle. As it has been observed, the general map or perfect order generating the highest production rate will be the total jigsaw the aggregate or ultimate picture -formed by partial jigsaw puzzles. That is the optimal menu. In this logical sequence, the total of the partial added value (it is not being called the total addition of added values) will be another jigsaw puzzle, the result of the addition of partial jigsaw puzzles. It is as if in a landscape, a jigsaw puzzle would define a volcano, another one the sea, another one palm trees, etc. The jigsaw puzzle of the added value would define a landscape resulting from the union of the partial images. If each image follows a rational, maximising criterion of production, the partial jigsaw puzzle, the addition of partial jigsaw puzzles, will necessarily imply the highest production rate from an order and technical point of view. To achieve the maximisation of this partial added value a suitable arrangement of the pieces that make it possible will be needed. And these pieces are, simultaneously, jigsaw puzzles that have been called unit production of the added value. In other words, to make the partial jigsaw explain the partial added value, it is also necessary to create some order among the unit jigsaw puzzles and choose, in the end, the optimal aggregate jigsaw puzzle. This means that the process of ordering continues incessantly; firstly, in order to combine factors and choose the optimal menu or jigsaw puzzle for each production, flour, for instance. And then each unit production of added values will be considered and put in order to generate the partial added value.

This includes partial added values that correspond to partial jigsaw puzzles. Domestic production will be calculated by integrating partial added values, but the mere addition of added values will not be enough. There must be a rational integrating criterion to make sense. This integrating criterion is the efficient order of partial jigsaw puzzles. Once again, a new order or combination appears to rationalise production. Partial jigsaw puzzles are ordered and the one that makes sense in the logic of the highest (more effective) production rate is the one that is chosen.

The highest possible production rate represents the best resource allocation from a sequencing point of view. It considers the map of the country as an integrating unit of partial landscapes, of each province, for instance. This is the optimal menu when all factors involved in the country and, therefore, all partial productions, partial added values, are considered.

The process has been followed continuously from the microeconomic analysis to the macroeconomic one: starting from the ordering of production factors to final production. The opposite reasoning would also be valid: in a market of perfect competition and efficiently allocated factors of production, the domestic product involves all 
production factors: primary and secondary factors, all correctly ordered. Any different order will result in a production volume lower in quantity and quality, which means that there will not be an efficient allocation of resources.

\section{SCHUMPETERIAN DYNAMIC ECONOMICS AND ENTROPY}

If Schumpeterian dynamic economics is considered as a system in constant evolution over time and as consumers' tastes change, as well as changes in techniques and industrial organisation - then the sense of combination of production factors will be studied in the framework of this assumption.

Two complementary effects will take place: the change in consumer's taste will cause positive changes in production. Changes in techniques and organisation in production causing increases by themselves will raise consumers' aggregate appetite. By acting jointly, their tastes will be reinforced and will cause destabilising and dynamic effects in the system. This effect is called energetic, entropic or heat releasing in physics and the same terms can be used in economics.

Changes in order during the process of seeking the best order or menu defined by the final jigsaw constitute entropic effects so far as they generate higher production rates. If these variations are found in orders included in a dynamic type of economy as described by Schumpeter, the entropic process will be more important. The most important thing will be finding out to what extent it affects a dynamic economy in causing change is the order of production and if it is mostly entropic.

In a dynamic economy, there are new production factors, higher amounts of these become available, and new goods are produced, and there are positive changes in technique, which totally alters the initial economic reality. There is a constant game of uncertainty because the map built, emanating from and reminding us of an initial map, is constantly changing. How can the pieces be arranged to form a general map (building the jigsaw puzzle) if the map is constantly changing? This means that building a jigsaw puzzle becomes difficult as the potential and theoretical figures is uncertain. On the other hand, the system is provided with positive energy to overcome these obstacles. Indeed entropy in the system is based in the mechanical process by which entropy is generated and it is dominated by the energetic forces in the system.

The rational explanation for any change in the economy is the enthusiasm and strength of consumers and producers. There is anxiety to consume greater quantities and to improve product quality. Entrepreneurs, on the other hand, are not only excited with new challenges and developments that produce profits (Schumpeterian profits) but also they are dogged by the threat of losses. Perfect competition brings about these consequences: not innovating is equal to disappearing. These are the endogenous energies that propel those that put the pieces in order and these actors try to dominate uncertainty in the system.

The jigsaw puzzle grows with new and different pieces and this entropic explosion causes uncertainty, but it is also true that producers will quickly put their pieces in order pushed by ambition, fear and their interest in progress.

In fact, there is neither static nor dynamic balance. The former is impossible by definition in a Schumpeterian dynamic economy. But dynamic balance is not possible either because, in the end, it comes from the tension between progress (new techniques, new factors, change in tastes, etc) and its product, that is uncertainty, and the importance of producers who try to control the fluid situation. The way to dominate the market or of building a dynamic puzzle is to increase efficiency in ordering the pieces in the same proportion or speed that pieces grow supposing there are scale economies in production. This assumption is difficult to explain in concept or figures.

A difficult question should now be answered. Is uncertainty - immeasurable uncertainty - equivalent to entropy, a measurable issue? The expansion of the economic universe is generated, besides, from growth in production, of factors and its combination, by the wide variety of internal and external changes in the system.

The jigsaw puzzle grows and spreads and above all the same underlying landscape and design of the jigsaw puzzle changes and it is precisely here where uncertainty appears. Building a jigsaw puzzle is a difficult task and it 
implies high-energy consumption. If the jigsaw puzzle also grows the difficulty increases as well. And it is an impossible task to make a jigsaw puzzle if the final picture is constantly changing. The maker faces constant uncertainty, a dynamic and not static uncertainty. This statement about dynamic and static uncertainty could probably be criticised by any economist since it is ambiguous to understand. It could be defined as follows: In a given economic situation, considering factors, technique, consumers' tastes, etc, this takes it far from Walrasian balance and makes domestic product increase and energy consumption in the system unknowable.

Now the measurability can be discussed. Uncertainty is not measurable by definition and entropy is measurable as a measure of energetic variation. How can these two points be harmonized? Entropy is measurable if it were possible to provide sufficient data to the model concerning the economic system by itself. But the system works incessantly with ever changing information that is impossible to measure in reality (though it is measurable in theory).

The work of the producer becomes hard and undefined but not inefficient. The market does not stop working: On the one hand there is an explosive ordering process in the combination of goods for the consumer, who will change its tastes and will try to maximise utility. On the other hand, changes in technique will imply that the fingers and hands of producers will be reactivated to continue building the jigsaw puzzle and new pieces will be added. And, what is more difficult to admit: the figure in the jigsaw puzzle will change.

This is in essence the way a Schumpeterian economy works where supply and demand attract each other trying to coordinate each other by centripetal forces as in any other system, but their centripetal energy, led by inertia, tends to pull them apart. In the Walrasian approach the information tends to balance, and the general Walrasian balance is the exhaustive information. In this case the jigsaw puzzle can be ordered following a defined map.

In a centrifugal moving away, information decreases in the same proportion as uncertainty increases. In this case, the jigsaw puzzle cannot be made because the map is unknown and uncertainty defines the system.

\section{CONCLUSION}

Working on the construction of a jigsaw puzzle, a series of pieces must be rationally put in order to define a map, the final image of the jigsaw puzzle. The analysis is developed in the context of the production theory and it is understood that the final jigsaw puzzle will determine a certain production volume. This is the methodological procedure.

At the same time, the universe of the jigsaw puzzle has been formed by combinations of production factors and more or less efficient combinations of the allocation of factors result from this action.

To utilise this approach, it is necessary to know the nature of combinations, menus -which are the pieces and the arrangement or final placement of all pieces that will form the final image.

Factors can be combined, the correct term would be ordered, in different ways. If all factors are considered, without repeating any of them, the sum of all possible orders is counted as $\mathrm{n}$ ! Then, all possible combinations are already known. Each combination is called a productive menu since each menu determines a specific measurable level of production and, obviously, orderable, in the case when there is only one menu that determines each production level. Each menu, generating a theoretical and real production level, is a piece of those that are going to be put in order. The rational integration of pieces (efficient allocation of resources) will shape the map of the final jigsaw puzzle.

Menus will be arranged on a scale or ladder of production levels in such a way that it is possible to establish a general map where all menus are included, considering criteria of efficiency in production. The general view of all menus is the final jigsaw puzzle. The general project of the jigsaw puzzle or the general guide map for arranging and placing the pieces is called the map of the jigsaw puzzle. This is a particular example of a jigsaw puzzle where the effort in building it is progressively being reduced as it is being made. If the yield in building a jigsaw puzzle were 
proportional to the number of units, and the progressive ease in building it yields a reduction of unitary costs, economies of scale can be obtained and determined.

There are very efficient subgroups of orders in production and, therefore, it is difficult to replace them by other orders. These are weldings. Weldings are groups of factors strongly linked and accepting their existence is effort saving when counting orders. They also imply cost-savings in building a jigsaw puzzle.

There can be agreements in building a jigsaw puzzle, and these agreements with combinations or weldings will cause some difficulties. Agreements lead to a common order that defines the common jigsaw puzzle.

Asymmetrical information, adverse selection and moral risk may appear when building a jigsaw puzzle, whenever there is a series of orders and the effort to order is known by some but unknown by others.

Dynamic economies, such as those described by Schumpeter, pose a real challenge to this approach since they consider uncertainty in the economic system. Domestic production grows, factors and quantity vary, etc. and, therefore, the arrangement in the map of the jigsaw puzzle changes. The threat to order grows because new pieces or factors are constantly being incorporated.

Suggestion for Future research: Jigsaw puzzle, map, order, menu, permutation, welding, hard-core, asymmetrical information, economic dynamics.

\section{AUTHORS NOTE}

My first research papers on the applications of the combinatorial theory in Microeconomics were published in 1993 and 1994. These articles were entitled: La Teoría Combinatoria Aplicada a la Teoría de la Utilidad and Teoría Combinatoria Aplicada a la Teoría de la Producción, respectively. This research, as the conceptual origin of other fields of research, diversified into issues such as the theory of chaos, social choice, asymmetrical information and this work on the building of jigsaw puzzles. These papers, among others, were: La Teoría Combinatoria en las Elecciones Públicas (2001), Elección Social, Valores sociales y Teoría Combinatoria (2002), Asimetría y Riesgo Moral en Leviathan indiferente ( 2003), Preferencias y Orden Combinatorio (2003), Simetrías en la Teoría combinatoria de Leviathan (2003), Caos y Orden Combinatorio en la Economía (2004), Una Teoría del Caos. Información Asimétrica en el Universo Combinatorio (2004). Combinatorial Theory Applied to the study of production (2004), Chaos and combinatorial Ordering in Economics: Chaos and System's Energy Are Similar Concepts (2004), Economy and Business-Business and the Economy (2004), Entropía, Caos y Teoría Combinatoria en la Economía, Business, Combinatorial Theory and Decision- Making (2005), Entropía: Caos Convergente y Caos Divergente (2005), Symmetric Combination With Leviathan combinatorial Theory Applied to Public Choice (2006).

\section{REFERENCES}

1. Arrow, J. K., (1950)A Difficult in the Concept of Social Welfare, Journal of Political Economy, 58.

2. $\quad$ Aumann Robert, J.(1987): Game Theory, in New Palgrave.

3. Backward Induction and Common Knowledge of Rationality, Games EB repeated Games of Incomplete Information, (1995) with M. Maschler.

4. $\quad$ Baumol, W.W. J., (1946) Community Indifference, Review of Economic Studies, 14.

5. Bergson, A., A., (1938): Reformulation of Certain Aspects of Wealfare Economics, Quaterly Journal of Economics, 52.

6. Binmore, Ken, (1999): Game Theory and Social Contract: Playing Fair, MIT Press.

7. Debreu, G., (1951) The Coeficient of Resource Allocation, in Ecta, 19, pp 273-92.

8. $\quad$ Dutta, Prajit, (1999): Strategies and Games: Theory and Practice, MIT Price.

9. Fisher, Ronald, (1930): The Genetical Theory of Natural Selection. Clarendon Press, Oxford.

10. Fudenberg, Drew y Jean Tirole(1991), Game Theory, MIT Press, ISBN 0262061414.

11. Friedman, J.W.(1991): Teoría de Juegos con Aplicaciones a la Economía. Editorial alianza Universidad.

12. Ginits, Herbert (2000): Game Theory Evolving. Princeton Univeristy Press, ISBN 0691009430. 
13. Harsanyi, J.C., (1953) Cardinal Utility in Welfare Economics and in the Theory of Risk Taking, Journal of Political Economy, 61.

14. Hicks, J.R., (1939): Value and Capital, Clarendon Press, Oxford.

15. Houthakker, H.S., (1950): Revealed Preference and the Utility Function, Econometrica 17.

16. Kreps, D.M.(1994) : Teoría de los Juegos y Modelación Económica. Fondo de cultura Económica, $1^{\circ}$ edición.

17. Majundar, T., (1956) Choice and Revealed Preference, Econometrica, 24.

18. Mishan, E.J., (1956) An Investigation into Some Alleged Contradictions in Welfare Economics, Economic Journal 67.

19. Morgestern, Oskar y John Von Neuman, (1947): The Theory of Games and Economic Behavior. Princeton Univeristy Press.

20. Nash, John, (1950): Equilibrium Points in n-person Games, Porceedings of the national Academy of the USA, 36(1):48-49.

21. Poundstone William: (2005): El Dilema del Prisionero, Alianza Editorial.Madrid.

22. Samuelson, P.A., (1947): Foundation of Economics Analysis, Harvard University Press, Cambridge, Mass.

23. Simon Herbert, (1987): Scientific Discovery: Computional Explorations of the Creative Processes, with Pat Langgley, Gary Bradshaww and Jan Zytkow, MIT Press.

24. Smith Maynard, John, (1982) : Evolution and the Theory of Game. Cambridge University Press.

25. Sen, A. K., (1963): Distribution, Transitivity and Little's Welfare Criteria, Economic Journal, 73.

26. Villacís, J., (1993): La Teoría Combinatoria Aplicada a la Teoría de la Utilidad. Esic Market, n 79. Madrid.

27. (1994): La Teoría Combinatoria Aplicada al Estudio de la Producción , Esic Market, pp 43-58.

28. (2004): Chaos and Combinatorial Ordering in Economics: Chaos and a Systems Energy Are Similar Concepts. Conference, $6^{\text {th }}$ August in Istambul.

29. (2005): Business, Combinatorial Theory and Decision Making, The Business Review, Cambridge,volume 3, December, pp 55-60. 


\section{NOTES}

